\title{
TEORES DE MICRONUTRIENTES NO SOLO E FOLIAR COM APLICAÇÃO DE FONTES QUELATADAS E SULFATADAS EM FEIJÃO
}

\author{
ADRIANE DE A. SILVA ${ }^{1}$, PEDRO A. COUTO JUNIOR ${ }^{2}$, ANGELA M. Q. LANA ${ }^{3}$, \\ REGINA M. Q. LANA ${ }^{4}$
}

\begin{abstract}
RESUMO: As fontes de micronutrientes apresentam, em geral, baixa disponibilidade no solo, e espera-se que o uso de fontes quelatadas aumente a absorção destes elementos. Objetivou-se comparar formulações de fertilizantes quelatizados e sulfatados, na absorção dos micronutrientes, zinco e cobre, com aplicação de diferentes doses na cultura do feijão. O experimento foi conduzido, na casa de vegetação da Universidade Federal de Uberlândia. Os fertilizantes utilizados foram fontes quelatadas e sulfatadas, nas doses de 0;20; 40; 60 e $80 \mathrm{mg} \mathrm{dm}^{-3}$. Instalaram-se, em vasos

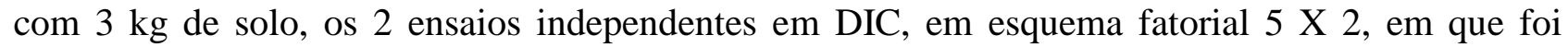
semeado feijão da variedade Pérola, sendo que, após 15 dias após emergência (DAE), realizou-se o desbaste, deixando então duas planta por vaso. A semeadura foi realizada no dia 09 de abril de 2010, e o corte das plantas foi realizado quando mais de $50 \%$ se encontravam no estágio R5. A aplicação com a fonte quelatada aumentou significativamente o teor de $\mathrm{Cu}$ disponível no solo e na planta. A fonte sulfatada não foi eficiente para fornecer $\mathrm{Cu}$ para o solo e para a planta. As fontes de Zn avaliadas não diferiram entre si na disponibilidade do solo, porém a absorção foi maior com o uso da fonte sulfatada.
\end{abstract}

PALAVRAS-CHAVE: Phaseolus vulgaris L., Cu e Zn, quelato EDTA.

\section{SOIL AND LEAF MICRONUTRIENT CONCENTRATION WITH THE APPLICATION OF SULFATE AND CHELATED SOURCES AND IN COMMON BEAN}

\begin{abstract}
The micronutrient sources usually have low availability in the soil, and it is expected that the use of chelated sources increase their absorption. The objective of this work was to compare chelated and sulfate fertilizer formulations at varied doses over common bean crop and their zinc and copper micronutrient absorption. The experiment was carried out under greenhouse in the Federal University of Uberlândia. The chelated sources and sulfate fertilizers were applied at 0 , 20, 40, 60 and $80 \mathrm{mg} \mathrm{dm}^{-3}$ doses. They were performed in duplicate $3 \mathrm{~kg}$ soil pots in a completely randomized design and a $5 \times 2$ factorial scheme. The "Pérola" variety was sown; and, in 15 days after emergence (DAE) there was thinning, leaving two plants per pot. The seeds were sown on April $9^{\text {th }}, 2010$ and plants were cut when more than 50\% was at R5 stage. The chelated source application significantly increased the available $\mathrm{Cu}$ content in the soil and plant. The sulfate source was not efficient to provide $\mathrm{Cu}$ neither to the soil nor to the plant. The $\mathrm{Zn}$ evaluated sources did not differ in its availability in soil, but its plant uptake was higher when using a sulfate source.
\end{abstract}

KEY WORDS: Phaseolus vulgaris L., $\mathrm{Cu}$ and Zn, EDTA chelate.

\footnotetext{
${ }^{1}$ Zootecnista, Doutora em produção Animal, Professora Adjunta do Instituto de Ciencias agrárias da Universidade Federal de Uberlândia-ICIAG-UFU, adriane @iciag.ufu.br

${ }^{2}$ Eng ${ }^{\text {O }}$ Agrônomo, Mestrando do Programa de Pós-Graduação em Agronomia do Instituto de Ciências Agrárias da Universidade Federal de Uberlândia, pj-dm@hotmail.com

${ }^{3}$ Eng $^{\mathrm{a}}$ Agrônoma, Professora Associada do departamento de Zootecnia da Universidade Federal de Minas Gerais, angelaquintao@gmail.com

${ }^{4}$ Eng $^{a}$ Agrônoma, Professora Titular do Instituto de Ciências agrárias da Universidade Federal de Uberlândia -ICIAG-UFU, rmqlana@iciag.ufu.br

Recebido pelo Conselho Editorial em: 22-9-2011
}

Aprovado pelo Conselho Editorial em: 14-10-2013 


\section{INTRODUÇÃO}

O feijão (Phaseolus vulgaris L.) é um dos mais importantes alimentos da dieta do brasileiro, por ser reconhecidamente uma excelente fonte de proteína, sendo o Brasil o maior produtor e consumidor mundial de feijão, com um consumo per cápita de $20,3 \mathrm{~kg} \mathrm{ha}^{-1}$. Também tem importância econômica e social, em razão de ser cultivado em grandes áreas e devido à mãodeobra que emprega durante o ciclo da cultura e em muitos casos também ser cultivado por agricultores familiares para atender à necessidade de subsistência.

Na região dos cerrados, com solos originalmente muito intemperizados, após a abertura das fronteiras agrícolas, observou-se que há uma grande demanda de aplicação de micronutrientes. Principalmente com o aumento de produtividade dessas áreas e as constantes exportações destes micronutrientes, começou a ser mais frequente o relato de sintomas de deficiência e resposta em produtividade com a aplicação de fontes de micronutrientes.

Há aumento de áreas com deficiência de micronutrientes, e esse fato deve-se a resistência dos produtores em aplicar fontes de micronutrientes (como os sulfatos e óxidos) em solos com baixa disponibilidade, pois muitas vezes essas aplicações não respondem às exigências das culturas, o que limita sua produção. Uma opção é a utilização de aplicações foliares, o que muitas vezes acaba sendo mais cara e não garantindo a aplicação de segurança desses nutrientes via solo, e mais recentemente o uso de fontes quelatadas (Cosmoquel) também têm sido utilizadas via solo. Essas alternativas tentam suprir a demanda das culturas, uma vez que o papel dos nutrientes é fundamental durante as fases de formação, desenvolvimento e maturação das sementes, principalmente na constituição das membranas e no acúmulo de carboidratos, lipídios e proteínas (SÁ, 1994).

RAIJ (2011) afirmou que a absorção de nutrientes pela planta depende da sua disponibilidade, a qual é influenciada por diversos fatores, dentre os quais, as formas químicas em que o nutriente é encontrado no solo, a capacidade de absorção da cultura, o desenvolvimento do sistema radicular, o tempo de crescimento e, ainda, as condições climáticas e da disponibilidade de outros nutrientes.

Alguns pesquisadores, (LOPES, 1984; ALVAREZ et al., 1999; FERREIRA et al., 2001), avaliando o teor de micronutrientes na região dos cerrados, determinaram os seguintes níveis críticos: $1,0 \mathrm{mg} \mathrm{dm}^{-3}$ para $\mathrm{Cu} ; 30 \mathrm{mg} \mathrm{dm}^{-3}$ para Fe; $5,0 \mathrm{mg} \mathrm{dm}^{-3}$ para $\mathrm{Mn} ; 1,0 \mathrm{mg} \mathrm{dm}^{-3}$ para $\mathrm{Zn}$.

MORAES et al. (2010) relatam que o uso de produtos que estimulam o crescimento vegetal em combinação com micronutrientes devem ser buscados com o intuito de aumentar a produtividade das culturas, e, ainda, formulações específicas de micronutrientes para uma determinada cultura e região serão cada vez mais requeridas.

As pesquisas referentes a micronutrientes são raras, comparativamente às investigações envolvendo o uso de macronutrientes; especificamente no caso do feijoeiro, poucos são os trabalhos que abordam o assunto, e, em muitos casos, com resultados contraditórios (TEIXEIRA et al., 2008). Segundo COSTA (2008), existe carência de trabalhos de pesquisa no Brasil avaliando a eficiência de quelatos ou complexos, principalmente quando aplicados no solo.

Sendo assim, o objetivo deste trabalho foi avaliar o efeito de fontes de micronutrientes sulfatadas e quelatadas, nos atributos químicos do solo, parâmetros vegetativos e teores de nutrientes foliares em feijão cv. Pérola em casa de vegetação.

\section{MATERIAL E MÉTODOS}

O experimento foi instalado na casa de vegetação da Universidade Federal de Uberlândia. Instalaram-se 2 ensaios independentes em delineamento inteiramente casualizados, em esquema fatorial 5 X 2 ( 5 doses e 2 fontes de micronutrientes).

No ensaio para avaliação de cobre, os tratamentos aplicados consistiram na aplicação do equivalente a $0 ; 20 ; 40 ; 60$ e $80 \mathrm{mg} \mathrm{dm}^{-3}$ de cobre e zinco, sendo as fontes sulfato cúprico P.A 
$(25,45 \%$ de $\mathrm{Cu})$ e Cosmoquel EDTA-Cu $(10 \%$ p/p) e as fontes sulfato de zinco P.A $(22,75 \%$ de $\mathrm{Zn})$ e Cosmoquel EDTA-Zn (10\% p/p).

Em ambos os ensaios, foi realizada a adubação de semeadura-padrão com o equivalente a 20 $\mathrm{kg}$ de $\mathrm{N}, 120 \mathrm{~kg}$ de $\mathrm{P}_{2} \mathrm{O}_{5}$ e $90 \mathrm{~kg}$ de $\mathrm{K}_{2} \mathrm{O}$.

O solo utilizado é um Latossolo Amarelo ácrico típico, A moderado, fase cerrado tropical subcadocifólio, relevo plano, com textura muito argilosa $\left(121 \mathrm{~g} \mathrm{~kg}^{-1}\right.$ de areia grossa, $69 \mathrm{~g} \mathrm{~kg}^{-1} \mathrm{de}$ areia fina, $24 \mathrm{~g} \mathrm{~kg}^{-1}$ de silte e $806 \mathrm{~g} \mathrm{~kg}^{-1}$ de argila), cujas características químicas se encontram na Tabela 1. Observa-se que este solo apresenta, de acordo com as classes de interpretação da disponibilidade para os micronutrientes CFSEMG (1999), uma média disponibilidade natural de cobre, e o zinco se encaixa-se em uma disponibilidade muito baixa.

TABELA 1. Caracterização do Latossolo Amarelo ácrico típico, antes da aplicação dos tratamentos, Uberlândia, 2010. Characterization of a typical yellow Latosol before the treatments, Uberlândia, 2010.

\begin{tabular}{|c|c|c|c|c|c|c|c|c|c|c|}
\hline $\mathrm{P}$ & $----\mathrm{mg} / \mathrm{dm}^{-3}--$ & $\mathrm{SO}_{4}$ & $\mathrm{Al}$ & $\mathrm{Ca}$ & $\begin{array}{r}\mathrm{Mg} \\
---\mathrm{cn} \\
\end{array}$ & $\begin{array}{c}\mathrm{H}+\mathrm{Al} \\
/ \mathrm{dm}^{-3}-\end{array}$ & $\begin{array}{r}\text { SB } \\
\end{array}$ & $\mathrm{T}$ & $\mathrm{V}$ & $\begin{array}{c}\mathrm{MO} \\
\mathrm{dag} / \mathrm{kg}\end{array}$ \\
\hline 9,5 & 34 & 4 & 0,4 & 0,4 & 0,1 & 4,30 & 0,59 & 4,89 & 12 & 2,6 \\
\hline $\mathrm{pH}$ & \multicolumn{2}{|c|}{ B } & \multicolumn{2}{|c|}{$\mathrm{Cu}$} & \multicolumn{2}{|c|}{$\mathrm{Fe}$} & \multicolumn{2}{|c|}{$\mathrm{Mn}$} & \multicolumn{2}{|c|}{$\mathrm{Zn}$} \\
\hline $\mathrm{H}_{2} \mathrm{O}$ & \multicolumn{10}{|c|}{ 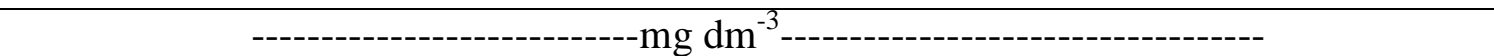 } \\
\hline 5,2 & \multicolumn{2}{|c|}{0,12} & \multicolumn{2}{|c|}{1,2} & & 56 & \multicolumn{2}{|c|}{1,2} & \multicolumn{2}{|c|}{0,4} \\
\hline
\end{tabular}

A semeadura foi realizada no dia 09 de abril de 2010, com quatro sementes de feijão da variedade Pérola, sendo que, após 15 dias após a emergência (DAE), realizou-se o desbaste, deixando então duas plantas por vaso. O experimento foi finalizado quando mais de $50 \%$ se encontravam no estágio R5 (emissão de botões florais), o que ocorreu em torno de 45 dias após a emergência.

Realizou-se a análise foliar da massa seca (MS) e ds teores no solo de micronutrientes, $\mathrm{Cu}$ e Zn, utilizando-se do extrator DTPA, de acordo com metodologia descrita em EMBRAPA (2009).

\section{RESULTADOS E DISCUSSÃO}

De acordo com a disponibilidade de cobre no solo (Figura 1), para a fonte de cobre quelatada com EDTA, observou-se que, a cada $1 \mathrm{mg} \mathrm{dm}^{-3}$ aplicado no solo, espera-se um acréscimo de 0,658 $\mathrm{mg} \mathrm{dm}^{-3}$ de cobre disponível no solo. O cobre com a fonte quelatada com EDTA apresentou efeito linear crescente, enquanto a fonte sulfatada não apresentou aumento de disponibilidade significativa $(\mathrm{p}<0,05)$. Pode-se afirmar que a fonte quelatada com EDTA apresenta uma disponibilidade do cobre para o solo superior à fonte sulfatada.

Na Figura 1, nota-se que, na testemunha, tem-se disponível 1,20 mg dm-3 .Quando se utilizou a fonte sulfatada, o teor obtido na dose de $80 \mathrm{mg} \mathrm{dm}^{-3}$ foi de $6,10 \mathrm{mg} \mathrm{dm}^{-3}$ e, nesta mesma dose, para fonte quelatada com EDTA, obtiveram-se $56,10 \mathrm{mg} \mathrm{dm}^{-3}$, ou seja, um incremento de $818 \%$ entre as fontes. 


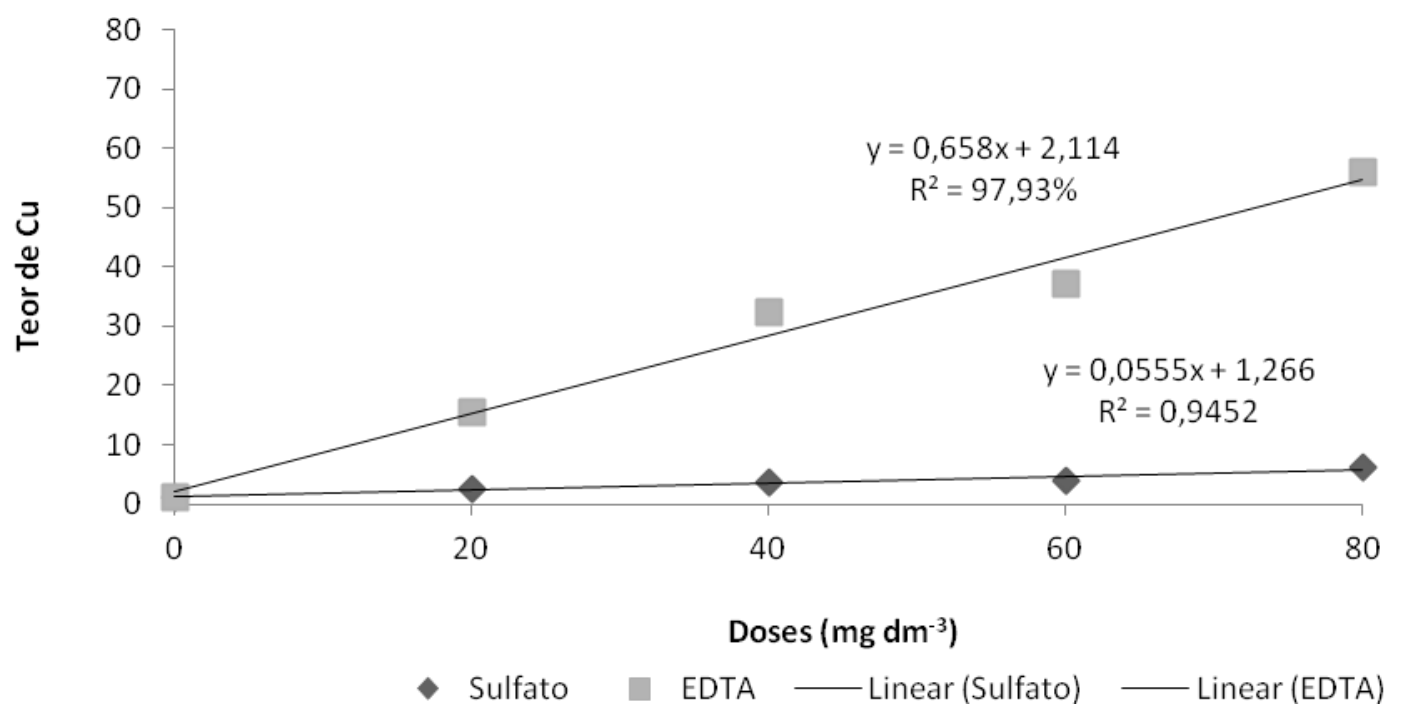

FIGURA 1. Teor de Cobre disponível no solo $\left(\mathrm{mg} \mathrm{dm}^{-3}\right)$ em um Latossolo Amarelo ácrico típico, fertilizado com fonte de cobre quelatada e sulfatada, Uberlândia, Minas Gerais,2010. Copper content available in soil $\left(\mathrm{mg} \mathrm{dm}^{-3}\right)$ in Yellow Latossol Typical fertilized with chelated and sulfated source of copper, Uberlândia, Minas Gerais, 2010.

Conforme ABREU et al. (2001), a deficiência do cobre é frequentemente observada em plantas crescendo em solos com baixo teor de cobre total ou em solos com alto teor de matéria orgânica, pois este elemento é complexado em formas orgânicas e insolúveis. Neste experimento, a fonte sulfatada pode ter sofrido maior complexação com a matéria orgânica, diferentemente da fonte quelatada que é protegida pelo EDTA. E isto indica que o uso de fonte quelatada, pode ser recomendada para o fornecimento deste elemento.

Nota-se que o valor avaliado da testemunha de $1,2 \mathrm{mg} \mathrm{dm}^{-3}$ encontra-se em uma classe média na interpretação da disponibilidade para micronutrientes (CFSEMG-1999), e que, independentemente da fonte usada, com qualquer uma das doses aplicadas, os valores encontramse com teor de disponibilidade bom ou muito bom (alto). Apesar de valor muito elevado de $\mathrm{Cu}$ com o uso da fonte quelatada, não se observaram sintomas de toxidez para as plantas. $\mathrm{O}$ que pode ter ocorrido é a necessidade de um ajuste da metodologia de análise deste elemento no solo, quando se utiliza uma fonte quelatada, pois com o uso do DTPA observou-se valor 70 vezes maior ao teor crítico superior descrito por DOURADO NETO \& FANCELLI (2000), que é de $0,8 \mathrm{mg} \mathrm{kg}^{-1}$; porém, observando-se a planta, não houve sintomas de toxidez.

VALE \& ALCARDE (1999) esclarecem os diferentes tipos de extratores utilizados para avaliação de micronutrientes e indicam que os agentes quelantes se mostraram também promissores para avaliar a disponibilidade de micronutrientes catiônicos no solo, pois podem combinar os metais livres em solução, formando complexos iônicos solúveis, reduzindo a atividade do metal no estado de máxima oxidação. RINGBOM (1963) relatou que os valores de $\mathrm{pH}$ em que as constantes de estabilidade do EDTA complexam $\mathrm{Cu}, \mathrm{Fe}, \mathrm{Mn}$ e $\mathrm{Zn}$ estão entre 9 e 10. Ou seja, as fontes já quelatadas, quando analisadas em DTPA, $\mathrm{pH} 7,3$, tendem a sofrer dissociação e a ficar totalmente disponíveis. Essas particularidades, quando se utiliza um fertilizante com tecnologia diferenciada, deve ser mais bem avaliada quanto ao método de avaliação, pois não são fontes convencionais muito estudadas. VALE \& ALCARDE (1999) concluíram que a garantia dos micronutrientes catiônicos pelo teor total, conforme exige a legislação, não indica a real disponibilidade dos micronutrientes nos fertilizantes.

Quando se correlacionam os níveis disponíveis no solo com a taxa de absorção da cultura, observa-se que a fonte quelatada foi mais eficiente em fornecer $\mathrm{Cu}$ do que a fonte sulfatada. De 
acordo com os teores foliares (Figura 2), observa-se que, mesmo com os altos teores no solo, somente a partir da dose de $40 \mathrm{mg} \mathrm{dm}^{-3}$ que a planta se apresentou dentro da faixa de suficiência proposta por MALAVOLTA et al. (1989). Já com a fonte sulfatada, a planta absorveu menos cobre do que a concentração observada na testemunha.

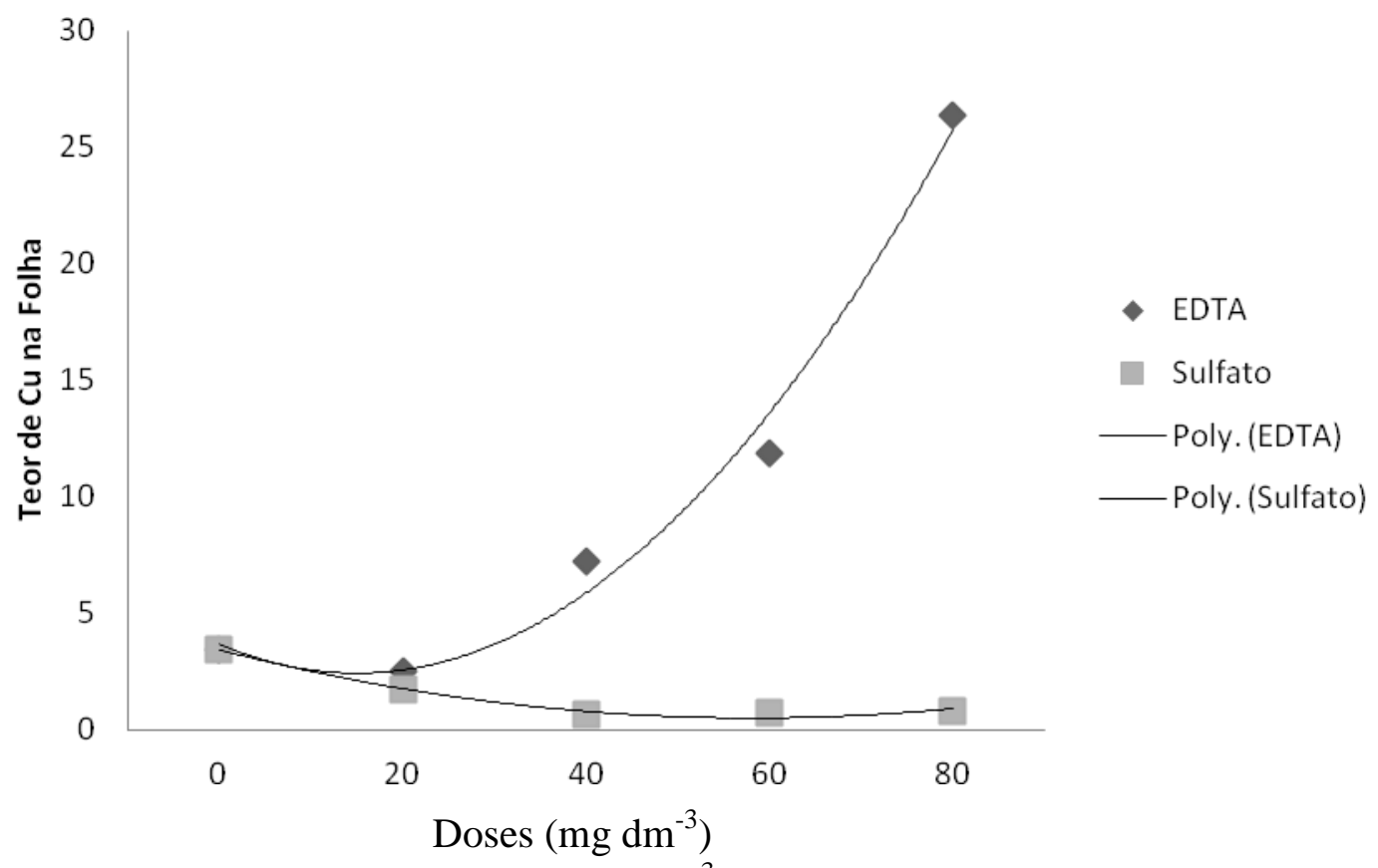

FIGURA 2. Teor de Cobre disponível na folha $\left(\mathrm{mg} \mathrm{dm}^{-3}\right)$ em um Latossolo Amarelo ácrico típico fertilizado com fonte de cobre quelatada e sulfatada, Uberlândia, Minas Gerais, 2010. Copper content available in leaf $\left(\mathrm{mg} \mathrm{dm}^{-3}\right)$ in Yellow Latossol Typical fertilized with chelated and sulfated source of copper, Uberlândia, Minas Gerais, 2010.

De acordo com a disponibilidade de cobre na folha (Figura 2), para a fonte de cobre quelatada com EDTA, observou-se que a mesma, na dose de $20 \mathrm{mg} \mathrm{dm}^{-3}$ aplicado no solo, não foi o suficiente, para que a planta suprisse sua necessidade de cobre, porém na dose de $40 \mathrm{mg} \mathrm{dm}^{-3}$ já corresponde a um nível suficiente de acordo com a análise de tecidos, na interpretação da disponibilidade para micronutrientes (CFSEMG, 1999),

Na Figura 2, nota-se que, na testemunha, encontram-se disponíveis $3,46 \mathrm{mg} \mathrm{dm}^{-3}$ de cobre. Quando se utilizou a fonte sulfatada, o teor obtido na dose de $20 \mathrm{mg} \mathrm{dm}^{-3}$ foi de $1,73 \mathrm{mg} \mathrm{dm}^{-3}$, inferior à testemunha, indicando que, quando se usa a fonte sulfatada de cobre, mesmo em doses mais elevadas, ela não tem uma disponibilidade ideal para a planta, e esse fato indica a baixa disponibilidade da fonte utilizada.

Os teores foliares variam entre espécies, parte das plantas e fatores ambientais e segundo ROMHELD \& MARSCHNER, (1991), esses teores variam entre 1 e $35 \mathrm{mg} \mathrm{kg}^{-1}$, sendo que, em alguns ecotipos, podem-se observar teores superiores a $100 \mathrm{mg} \mathrm{kg}^{-1}$. Porém, a variação observada foi em função da fonte utilizada e não em função das espécies ou parte da planta amostrada.

Segundo MORTVEDT (1999), a eficiência relativa, para as culturas, dos quelatos aplicados ao solo pode ser de duas a cinco vezes maiores por unidade de micronutriente do que as fontes inorgânicas, enquanto o custo do quelato por unidade de micronutriente pode ser de cinco a cem vezes mais altas. Esse aspecto constitui-se em uma limitação ao uso desses produtos para culturas de baixo valor agregado, principalmente quando se observa que não houve ganho em produção de MS, como observado neste experimento. As fontes quelatadas, hoje disponíveis no mercado, têm um valor superior a fontes convencionais, mas a maior disponibilidade é um fator de convencimento para sua aquisição. 
Para a cultura do feijão devem-se realizar mais pesquisas com aplicação de micronutrientes, pois de acordo com DOURADO NETO \& FANCELLI (2000), o não atendimento do nível crítico reduz o rendimento da cultura. JUNQUEIRA NETTO (1997), estudando a extração total do feijoeiro em um sistema de produção com $2.100 \mathrm{~kg}$ por hectare, observou que, em um hectare, a cultura exporta $30 \mathrm{~g} \mathrm{ha}^{-1}$ de cobre.

A produtividade de MS da parte aérea do feijão não apresentou efeito para as diferentes doses com o uso da fonte sulfato, somente com o uso da fonte EDTA (Figura 3). Observou-se massa seca decrescente com o aumento das doses aplicadas, podendo-se atribuir esse fato ao aumento de $\mathrm{Cu}$ (Figura 2), que pode indicar um início de toxidez na planta, pela maior absorção deste nutriente fornecido com o uso da fonte quelatada.

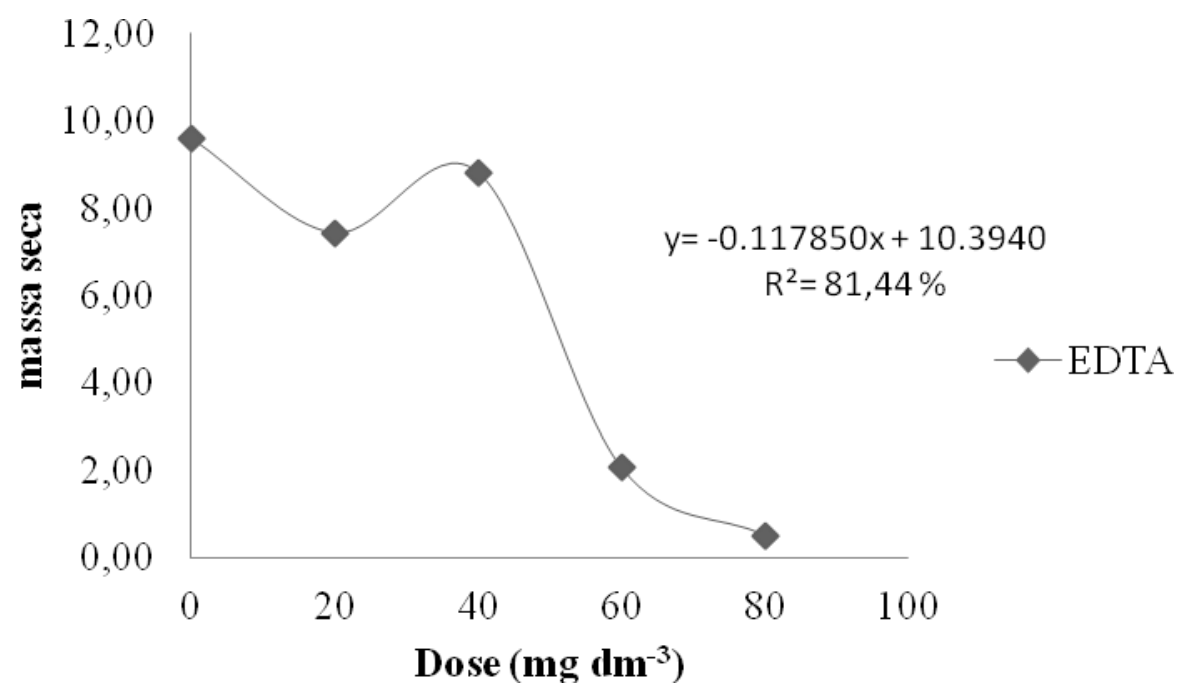

FIGURA 3. Produção de massa seca (MS) do feijão fertilizado com fonte de cobre quelatado, Uberlândia, Minas Gerais, 2010. Dry matter (DM) production in common bean fertilized with chelated and sulfated source of copper, Uberlândia, Minas Gerais, 2010.

Observa-se que somente houve variação entre as fontes aplicadas na produção de massa seca na dose de $80 \mathrm{mg} \mathrm{dm}^{-3}$, em que, com a aplicação de sulfato, a produção de massa seca foi $95 \%$ superior a obtida com a fonte EDTA (Tabela 2). Pode-se atribuir esse fato à maior absorção do elemento quando se utiliza uma fonte de maior disponibilidade, como a EDTA, e a redução na produção de massa seca começou a ser reduzida a partir da dose de $60 \mathrm{mg} \mathrm{dm}^{-3}$, indicando que essas doses podem ter promovido uma fitotoxidez.

TABELA 2. Massa seca da parte aérea de feijão-pérola fertilizado com fonte de cobre quelatado e sulfatado, Uberlândia, Minas Gerais, 2010. Dry Matter in common bean fertilized fertilized with pearl source of copper chelated and sulfated, Uberlândia, Minas Gerais, 2010.

\begin{tabular}{ccc}
\hline & & Fonte \\
\cline { 2 - 3 } Dose $\left(\mathrm{mg} \mathrm{dm}^{-3}\right)$ & EDTA & Sulfato \\
\hline 0 & $9,60 \mathrm{a}$ & $9,60 \mathrm{a}$ \\
20 & $7,43 \mathrm{a}$ & $6,49 \mathrm{a}$ \\
40 & $8,82 \mathrm{a}$ & $6,90 \mathrm{a}$ \\
60 & $2,06 \mathrm{a}$ & $11,19 \mathrm{a}$ \\
80 & $0,50 \mathrm{~b}$ & 39,23 \\
CV & 5,02 & \\
\hline
\end{tabular}

Letras diferentes na linha diferem entre si na linha, pelo teste de Tukey $(0,05)$ 
Observa-se (Figura 4) que não houve diferença entre as fontes aplicadas (quelatadas e sulfatadas), houve um aumento linear dos teores de $\mathrm{Zn}$ no solo, em função do aumento das doses. Observa-se que, no tratamento sem aplicação de $\mathrm{Zn}$, o elemento encontrava-se classificado com muito baixa disponibilidade no solo. Na dose de $20 \mathrm{mg} \mathrm{dm}^{-3}$, o teor observado estava classificado como muito elevado, ou seja, $>2,2 \mathrm{mg} \mathrm{dm}^{-3}$. Ambas as fontes demonstraram alta eficiência no fornecimento de $\mathrm{Zn}$.

COSTA (2008) verificou que, independentemente da fonte de $\mathrm{Zn}$ utilizada (sulfato de zinco, Zn-EDTA e Zn Lignosulfonato-LS) em um Latossolo Vermelho distrófico, textura média, os teores desse micronutriente no solo não apresentaram diferença, porem ocorreu aumento nos teores de $\mathrm{Zn}$ com o aumento das doses aplicadas. SLATON et al. (2005) também verificaram que não houve diferença entre a aplicação da fonte orgânica ( $\mathrm{Zn}-\mathrm{LS})$ e da inorgânica $\left(\mathrm{ZnSO}_{4}\right)$.

O zinco é um nutriente que de acordo BAR-YOSEF et al. (1980), tem grande adsorção com os constituintes da fase sólida, ou seja, ele fica adsorvido à CTC do solo; nesse sentido, a aplicação via sulfato permitiu que o nutriente esteja disponível para a planta. Ou seja, a fonte quelatada reduz a adsorção dos elementos, mas, neste caso, como o $\mathrm{Zn}$ tem afinidade com os coloides e é desejável que ele fique trocável, não se observou diferença significativa com o uso desta fonte.

JUNQUEIRA NETTO (1997), estudando a extração total do feijoeiro em um sistema de produção com $2.100 \mathrm{~kg}$ por hectare, observou que, em um hectare, a cultura exporta $80 \mathrm{~g} \mathrm{ha}^{-1} \mathrm{de}$ Zn. Observando o teor disponível de Zn no solo (Figura 4), independentemente da fonte aplicada, quelatada ou sulfatada, não houve diferença significativa entre as duas. No entanto, já na análise foliar (Figura 4), observa-se que a absorção pelas plantas foi diferenciada, e a fonte sulfatada obteve melhor disponibilidade do nutriente para as plantas.

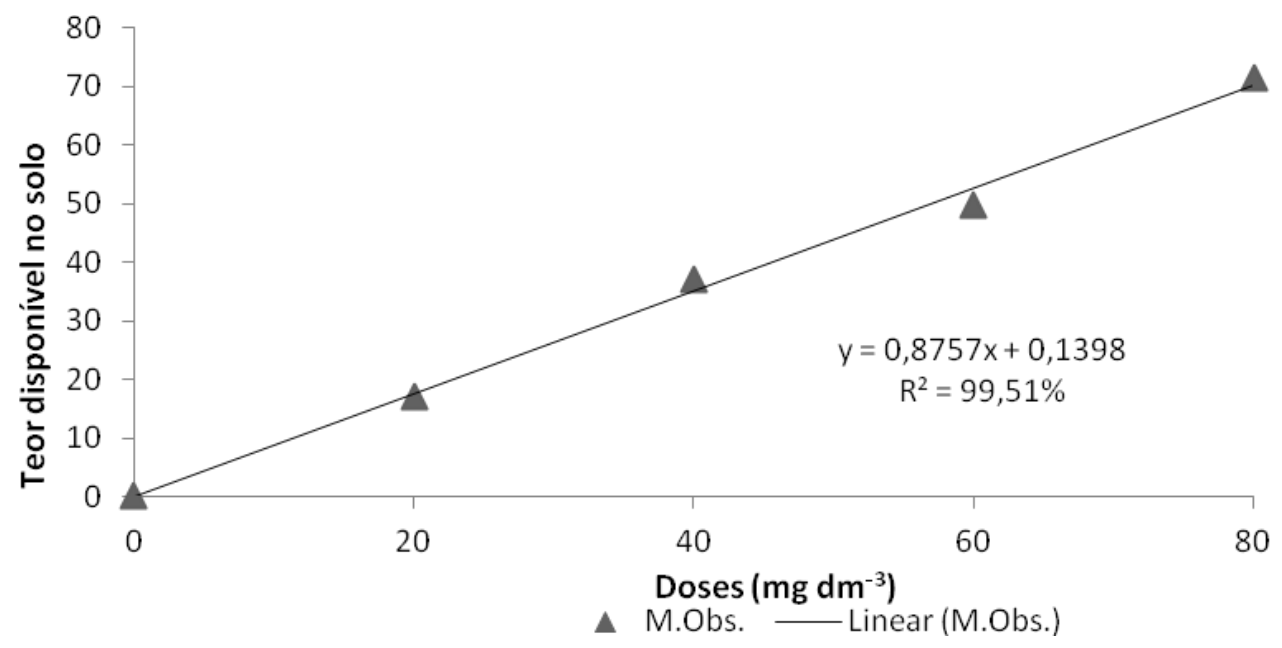

FIGURA 4. Teor de Zinco disponível no solo $\left(\mathrm{mg} \mathrm{dm}^{-3}\right)$ em um Latossolo Amarelo ácrico típico fertilizado com fonte de zinco quelatada e sulfatada, Uberlândia, Minas Gerais, 2010. Zinc content available in soil $\left(\mathrm{mg} \mathrm{dm}^{-3}\right)$ in Yellow Latossol Typical fertilized with chelated and sulfated source of copper, Uberlândia, Minas Gerais, 2010.

A disponibilidade de zinco na folha (Figura 5), independentemente da fonte aplicada, até à dose de $40 \mathrm{mg} \mathrm{dm}^{-3}$, encontra-se dentro da taxa de suficiência da cultura do feijão; porém, já na dose de $60 \mathrm{mg} \mathrm{dm}^{-3}$, o teor obtido corresponde a um nível elevado de acordo com a análise de tecidos na interpretação da disponibilidade para micronutrientes (CFSEMG,1999).

De acordo com COSTA (2008), a fonte Zn-LS proporcionou concentrações de Zn na planta de milho superiores à fonte sulfato de zinco. Os dados obtidos mostram, entretanto, que não houve 
diferença significativa entre a fonte quelatada (Zn-EDTA) e o complexo orgânico (Zn-LS) e entre o Zn-EDTA e a fonte sulfato de zinco.

Conforme MALAVOLTA et al. (1989), os teores adequados de zinco para a cultura do feijoeiro se encontra-se entre 20 e $100 \mathrm{mg} \mathrm{kg}^{-1}$; neste caso, estes autores indicam uma faixa mais ampla, em que as doses até $80 \mathrm{mg} \mathrm{dm}^{-3}$, em ambas as fontes, atenderiam à exigência da cultura.

Allen e Ternan (1966) afirmaram que o $\mathrm{ZnSO}_{4}$ (sulfato de $\mathrm{Zn}$ ) foi uma fonte satisfatória em experimento de casa de vegetação. Dados de campo com feijoeiro (Phaseolus vulgaris, L.) mostraram que $\mathrm{ZnO}$ granulado foi ineficiente como fonte de Zn (JUDY et al., 1964). Já neste experimento, observou-se que ambas as fontes testadas apresentaram absorção crescente até a dose de $60 \mathrm{mg} \mathrm{dm}^{-3}$.

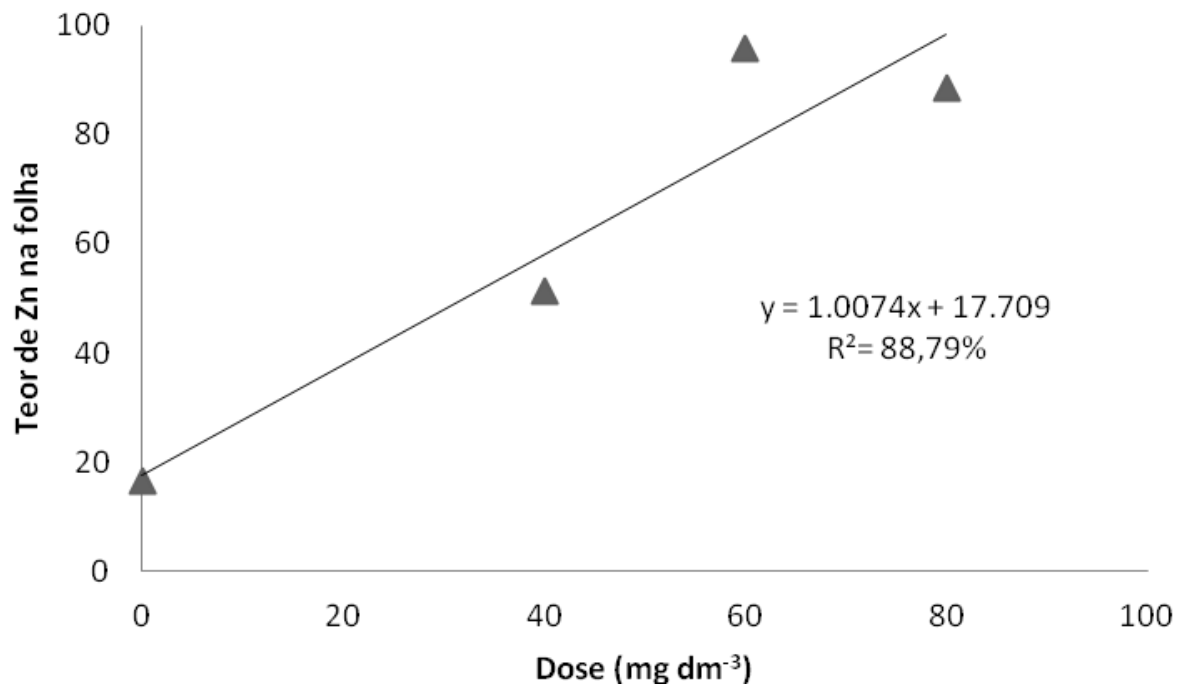

FIGURA 5. Teor de Zinco disponível na folha $\left(\mathrm{mg} \mathrm{dm}^{-3}\right)$ em um Latossolo Amarelo ácrico típico fertilizado com fonte de cobre quelatada e sulfatada, Uberlândia, Minas Gerais, 2010. Zinc content available in leaf $\left(\mathrm{mg} \mathrm{dm}^{-3}\right)$ in Yellow Latossol Typical fertilized with chelated and sulfated source of copper, Uberlândia, Minas Gerais, 2010.

Observa-se, na Tabela 3, que houve variação entre as fontes aplicadas, sendo que, para fonte sulfatada, houve incremento de MS, de 5\% em relação à fonte EDTA.

TABELA 3. Médias da massa seca da parte aérea de feijão-pérola fertilizado com fonte de zinco quelatado e sulfatado, Uberlândia, Minas Gerais, 2010. Dry Matter in common bean fertilized fertilized with pearl source of zinc chelated and sulfated, Uberlândia, Minas Gerais, 2010.

\begin{tabular}{cc}
\hline & Médias \\
\cline { 2 - 2 } Fontes & Massa Seca \\
\hline Edta & $61,54 \mathrm{~B}$ \\
Sulfato & $65,01 \mathrm{~A}$ \\
\hline CV $(\%)$ & 5,27 \\
\hline
\end{tabular}

Letras distintas diferem entre si pelo teste de Tukey $(0,05)$

COSTA (2008), estudando o comportamento das fontes de Zn (sulfato de zinco, Zn-EDTA e Zn-LS) na produção de massa seca do milho, observou que não ocorreram diferenças significativas entre as mesmas. Conforme também observado por SLATON et al. (2005), é importante relatar que nem sempre diferenças nas concentrações de $\mathrm{Zn}$ na planta, atribuídas às fontes desse micronutriente, garantirão comportamento distinto das mesmas em termos de produção de massa seca. 


\section{CONCLUSÃO}

A aplicação de cobre, com a fonte quelatada, aumentou significativamente o teor de $\mathrm{Cu}$ disponível no solo e na planta. Houve diferença significativa entre as fontes avaliadas, sendo que a sulfatada não foi eficiente para fornecer $\mathrm{Cu}$ para o solo e para a planta.

As fontes de zinco não diferiram entre si na disponibilidade no solo; porém, nas plantas, a absorção foi maior com o uso da fonte sulfatada.

A disponibilidade entre os micronutrientes e a absorção pelas plantas variaram de acordo com a fonte aplicada e o incremento das doses.

\section{REFERÊNCIAS}

ABREU, C.A. Disponibilidade e avaliação dos elementos catiônicos: zinco e cobre, In: FERREIRA, M.E.; CRUZ, M.C.P da; RAIJ, B. v.; ABREU, C.A. Micronutrientes e elementos tóxicos na agricultura. Jaboticabal, CNPq; FAPESP; Potafós, 2001. p.124-150.

ALLEN, S. E.; TERMAN, G. L. Response of maize and sudangrass to zinc in granular micronutrients. In: INTERNATIONAL SOCIETY OF SOIL SCIENCE. Transactions. Aberdeen: ISSS, 1966. p.255-266.

ALVAREZ, V,V.H.; NOVAIS,R.F.; BARROS,N.F. de. et al. Interpretação dos Resultados das Análises de Solo In: COMISSÃO DE FERTILIDADE DO SOLO DO ESTADO DE MINAS GERAIS. Recomendações para o uso de corretivos e fertilizantes em Minas Gerais: $5^{\mathrm{a}}$ aproximação. Viçosa, 1999. 359 p.

BAR-YOSEF, B.; FISHMAN, S.; TALPAZ, H. A model of zinc movement to single roots in soils. Soil Science Society of America Journal, Madison, v.44, p.1.272-1.279, 1980.

CFSEMG - Comissão de Fertilidade do Solo do Estado de Minas Gerais. Recomendações para o uso de corretivos e fertilizantes em Minas Gerais: $5^{a}$ aproximação. Viçosa, 1999. 359 p.

COSTA, R. S.S. Aplicação de quelatos de zinco em um solo deficiente cultivado com milho em casa de vegetação. 2008. 31f. Dissertação (Mestrado em Agronomia - Produção Vegetal) - Faculdade de Ciências Agrárias e Veterinárias, Universidade Estadual Paulista, Jaboticabal, 2008.

DOURADO NETO, D.; FANCELLI, A.L. Nutrição, adubação e calagem. In: DOURADO NETO, D.; FANCELLI, A.L. (Ed.). Produção de feijão. Guaíba: Livraria e Editora Agropecuária, 2000. p.49-84.

EMBRAPA - Empresa Brasileira de Pesquisa Agropecuária. Manual de análises químicas de solos, plantas e fertilizantes - 2. ed. rev. ampl. Brasília-DF : Embrapa Informação Tecnológica, 2009.

FERREIRA, M.E,; CRUZ, M.C.P. de; RAIJ, B.V. Micronutrientes e elementos tóxicos na agricultura. Jaboticabal: CNPq/FAPESP/POTAFOS, 2001.

JUDY, W.; LESSMAN, G.; ROZYCKA, T.; ROBERTSON, L.; ELLIS, B. G. Field and laboratory studies with zinc fertilization of pea beans. Quartely Bulletin. Michigan State University Agricultural Experiment Station, East Lansing, v. 46, p. 386-400, 1964.

JUNQUEIRA NETO, A. Micronutrientes na cultura do feijão. In: FANCELLI, A.L; DOURADO NETO, D. (ed.) Tecnologia da produção do feijão irrigado. Piracicaba: USP, ESALQ, 1997. 158 p.

LOPES, A.S. Micronutrientes nos solos e culturas brasileiras. In: SEMINÁRIO SOBRE FÓSFORO, CÁLCIO, MAGNÉSIO, ENXOFRE E MICRONUTRIENTES, 1984, São Paulo. Anais... São Paulo: MANAH, 1984. p.110-141.

MALAVOLTA, E.; VITTI, G.C.; OLIVEIRA, S.A. Avaliação do estado nutricional de plantas: princípios e aplicações. Piracicaba: Potafós, 1989. 208 p. 
MORAES, M. F.; ABREU JUNIOR, C. H.; LAVRES JUNIOR, J. Micronutrientes. In: PROCHNOW, L. I.; CASARIN, V.; STIPP, S. R. Boas práticas para uso eficiente de fertilizantes. Piracicaba: International Plant Nutrition Institute, 2010, p. 207-278.

MORTVEDT, J. J. Tecnologia e produção de fertilizantes com micronutrientes. Presença de elementos tóxicos. In: SIMPÓSIO SOBRE MICRONUTRIENTES E ELEMENTOS TÓXICOS NA AGRICULTURA, 1999, Jaboticabal. 1999.11p.

RAIJ, B. van. Fertilidade do solo e manejo de nutrientes. Piracicaba: International Plant Nutrition Institute, 2011, 420p.

RINGBOM, A. Complexation in analytical chemistry. New York: Interscience Publishers, 1963. 395 p.

ROMHELD, V.; MARSCHNER. H. Functions of micronutrients in plants. In: MORTVEDT, J.J., COX, F.R.; L.M. SHUMAN, R.M.; WELCH. R. M. (Ed.). Micronutrients in Agriculture. Madison Wisconsin: SSSA, 1991.

SÁ, M.E. Importância da adubação na qualidade de semente.In: SÁ, M.E.; BUZZETI, S. (Ed.). Importância da adubação na qualidade dos produtos agrícolas. São Paulo: Ícone, 1994.p.65-98.

SLATON, N.A.; GBUR JUNIOR, E.E.; WILSON JUNIOR, C.E.; NORMAN, R.J. Rice response to granular zinc sources varying in water-soluble zinc. Soil Science Society of America Journal, Madison, v.69, p. 443-454, 2005.

TEIXEIRA, I. R.; BORÉM, A.; SILVA, A. G. da; KIKUTI, H. Fontes e doses de zinco no feijoeiro cultivado em diferentes épocas de semeadura. Acta Scientrarum Agronomy. Maringá, v. 30, n. 2, p. 255-259, 2008.

VALE, F.; ALCARDE, J.C.; Solubilidade e disponibilidade dos micronutrientes em fertilizantes, Revista Brasileira de Ciência do Solo, Viçosa, MG, v. 23, p. 441-451, 1999. 